\title{
Review
}

International Archives of
Allergy
Immunology

\section{Differences in Reporting the Ragweed Pollen Season Using Google Trends across 15 Countries}

\author{
Jean Bousquet ${ }^{a, b, c}$ loana Agached Uwe Bergere Karl-Christian Bergmann ${ }^{\text {f, } g}$ \\ Jean-Pierre Besancenot ${ }^{\mathrm{h}}$ Philippe J. Bousquet ${ }^{\mathrm{i}}$ Tom Casale $^{\mathrm{j}}$

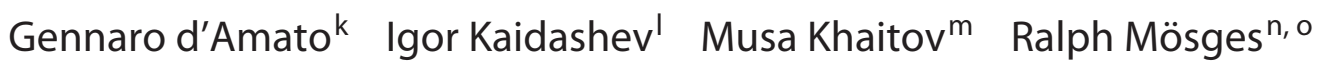 \\ Kristof Nekam $^{p}$ Gabrielle L. Onorato ${ }^{a}$ Davor Plavec ${ }^{q}$ r $\quad$ Aziz Sheikh $^{\mathrm{s}}$ \\ Michel Thibaudon $^{\mathrm{h}}$ Robert Vautard $^{\mathrm{t}}$ Mihaela Zidarn $^{\mathrm{u}}$
}

${ }^{a}$ MACVIA-France, Contre les Maladies Chroniques pour un Vieillissement Actif en France, European Innovation Partnership on Active and Healthy Ageing Reference Site, Montpellier, France; 'bINSERM U 1168, VIMA: Ageing and Chronic Diseases Epidemiological and Public Health Approaches, Villejuif, France; ${ }^{\circ}$ Université Versailles St-Quentin-

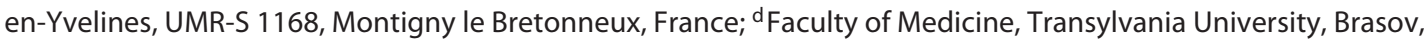
Romania; ' Department of Oto-Rhino-Laryngology, Aerobiology and Pollen Information Research Unit, Medical University of Vienna, Vienna, Austria; ${ }^{f}$ Comprehensive Allergy-Centre-Charité, Department of Dermatology and Allergy, Charité - Universitätsmedizin Berlin, Berlin, Germany; ${ }^{9}$ Global Allergy and Asthma European Network (GA2LEN), Berlin, Germany; ${ }^{\text {h}}$ RNSA (Réseau National de Surveillance Aérobiologique), Brussieu, France; ${ }^{\text {EEPAR }}$ U707 INSERM and EPAR UMR-S UPMC, Paris VI, Paris, France; 'Division of Allergy/Immunology, University of South Florida, Tampa, FL, USA; 'Division of Respiratory and Allergic Diseases, Hospital 'A Cardarelli', University of Naples Federico II, Naples, Italy; 'Ukrainina Medical Stomatological Academy, Poltava, Ukraine; ' ${ }^{2}$ Laboratory of Molecular Immunology, National Research Center, Institute of Immunology, Federal Medicobiological Agency, Moscow, Russian Federation; ${ }^{\mathrm{n}}$ Institute of Medical Statistics and Computational Biology Medical Faculty, University of Cologne, Cologne, Germany; ${ }^{\circ} \mathrm{CRI}$ - Clinical Research International Ltd, Hamburg, Germany; ${ }^{\mathrm{P} H o s p i t a l}$ of the

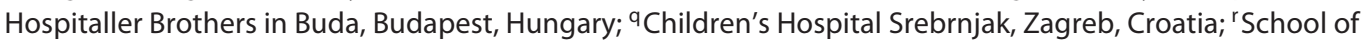
Medicine, University J.J. Strossmayer, Osijek, Croatia; ${ }^{5}$ Centre of Medical Informatics, Usher Institute of Population Health Sciences and Informatics, The University of Edinburgh, Edinburgh, UK; ${ }^{\mathrm{t} L S C E / I P S L}$, Laboratoire CEA/CNRS/ UVSQ, Gif-sur-Yvette, France; "University Clinic of Respiratory and Allergic Diseases, Golnik, Slovenia

\section{Keywords}

Allergy · Google Trends · Hay fever · Pollen · Ragweed · Rhinitis

\section{Abstract \\ Background: Google Trends (GT) searches trends of specific queries in Google, which potentially reflect the real-life epi- demiology of allergic rhinitis. We compared GT terms related to ragweed pollen allergy in American and European Union}

countries with a known ragweed pollen season. Our aim was to assess seasonality and the terms needed to perform the GT searches and to compare these during the spring and summer pollen seasons. Methods: We examined GT queries from January 1, 2011, to January 4, 2017. We included 15 countries with a known ragweed pollen season and used the standard 5-year GT graphs. We used the GT translation for all countries and the untranslated native terms for each coun-

Edited by: V. Niederberger-Leppin, Vienna.

\section{KARGER}

(c) 2018 S. Karger AG, Basel

E-Mail karger@karger.com

www.karger.com/iaa
Correspondence to: Prof. Jean Bousquet

CHU Arnaud de Villeneuve

371 Avenue du Doyen Gaston Giraud

FR-34295 Montpellier Cedex 5 (France)

E-Mail jean.bousquet@ orange.fr 
try. Results: The results of "pollen," "ragweed," and "allergy" searches differed between countries, but "ragweed" was clearly identified in 12 of the 15 countries. There was considerable heterogeneity of findings when the GT translation was used. For Croatia, Hungary, Romania, Serbia, and Slovenia, the GT translation was inappropriate. The country patterns of "pollen," "hay fever," and "allergy" differed in 8 of the 11 countries with identified "ragweed" queries during the spring and the summer, indicating that the perception of tree and grass pollen allergy differs from that of ragweed pollen. Conclusions: To investigate ragweed pollen allergy using GT, the term "ragweed" as a plant is required and the translation of "ragweed" in the native language needed.

(c) 2018 S. Karger AG, Basel

\section{Introduction}

For patients allergic to pollen, knowledge regarding the onset and duration of the pollen season is of great importance [1-3]. It is therefore essential to be able to forecast the onset of the pollen season as well as to characterise seasons in different places. Sentinel networks offer the potential to meet this need [4-7]. Pollen counts can forecast the exposure to pollen [8]. The assessment of allergen content in the air is feasible [9], but requires sophisticated methods that may not account for all of the pollen species in the ambient air. Furthermore, measurements at the individual level are challenging and expensive, although feasibility has been demonstrated through some wearable devices. Meteorological data may, in the future, be of interest when it comes to predicting the onset of the season [10]. However, more data are required. Combining several sources of information using advanced data engineering may also be important, but the data generated are complex and not yet available for all pollen species $[4-7,11]$.

Since forecasting remains a limited option, real-time epidemiological monitoring is an alternative. Google Trends (GT) is a web-based surveillance tool that uses aggregated Google searches to summarise the searching trends of specific queries. Recent studies have suggested the utility of GT for assessing the seasonality of allergic diseases [12-17] and for detecting influenza epidemics [18]. GT data may closely reflect the real-world epidemiology of allergic rhinitis (AR) and could potentially be used as a monitoring tool for AR [19]. However, besides the translation of the terms in different languages, cultural differences and different methods of accessing knowledge exist between countries and the same terms are not used similarly in all countries [20]. Moreover, for the development of a pan-European sentinel network, these terms should be clearly defined [1].

Ragweed is a highly allergenic pollen and its expansion in several countries is inducing increasing health issues [21]. The present study was conceived as a preliminary step to explore the use of GT in the epidemiological surveillance of AR. We sought to assess whether there were differences in the Google searches of AR and related topics for ragweed pollen allergy. We analysed multiple time series of Google search data on rhinitis, allergy, and pollen in Europe and North America from January 1, 2011, to January 4, 2017, with a focus on ragweed pollen allergy. The specific aims of the study were: (i) to assess whether the GT terms could accurately report both the onset of symptoms and of the ragweed pollen season accurately, (ii) to examine whether the GT translation of "ragweed" could be used without assessing the term in the native language, and (iii) to compare the seasonality of queries in American and European countries.

\section{Methods}

\section{GT Terms}

GT, a public web facility of Google Inc. based on Google Search, shows how often a particular search term is entered relative to the total search volume across various regions of the world and in various languages. The horizontal axis of the main graph represents time (starting from 2004), and the vertical axis shows how often a term is searched relative to the total number of searches globally (https://trends.google.com).

The following terms were used: "rhinitis," "allergic rhinitis," "hay fever," "pollen," "allergy," and "asthma" (disease and term). However, only the following 3 terms were analysed: "allergy," "hay fever," and "pollen," since "rhinitis" as a term or "hay fever" were labelled as "allergic rhinitis as a disease." "Asthma" and "conjunctivitis" were not selected as we found no seasonality associated with pollen in previous analyses [unpubl. observation]. Only "diseases" and "subjects" are translated by Google Translator (https:// translate.google.com), whereas "terms" or "terms of research" are not translated directly. The GT terms for ragweed pollen include "gender," "plant," and "subject." We tested the 3 terms and "gender" was the term needed to assess the ragweed pollen season in all countries tested. Moreover, we used "ragweed" as a GT translation and with the native languages of the countries studied.

In GT, data are normalised to the highest number of searches for each topic. A score of 100 meant that, on that day, the topic had the highest number of searches over the reference period included in the search ( 5 years in the present study) in that particular place. Comparisons across different areas should therefore be considered with care since the score in each area depends on the series of searches during the reference period in that area. Moreover, a high level of search (e.g., "allergy") reduces the level of the other searches. To account for this issue, we performed searches that included and excluded "allergy." 
Table 1. Patterns of countries during the spring and summer pollen seasons

\section{Pattern}

A Peak of "hay fever" greater than "allergy"

B Peak of "hay fever," "pollen," and "allergy"

C Peak of "pollen"

D Peak of "allergy"

E Peak of "allergy" and "hay fever"

F No season identified

G Peak of "allergy" and "pollen"

\section{Selection of Countries}

Countries with a population with a known allergy to ragweed pollen were studied (online suppl. Table 1; for all online suppl. material, see www.karger.com/doi/10.1159/000488391). They were European countries defined in a recent paper [22], former Yugoslavian countries [23], Canada, Russia, the USA, and Ukraine [24].

\section{Analyses}

We used the 5-year GT graphs to examine GT queries from January 1, 2011, to January 4, 2017, in American and European countries with a known ragweed pollen season. A visual analysis was carried out (J.B.) to assess the seasonality of ragweed pollen allergy.

Cultural differences exist between countries. Terms are therefore not used in the same way from one country to the next [20]. We compared the country patterns (based on seasons) with the mean 5 -year terms provided by GT, slightly modified from a previous paper in European countries (Table 1) [20]. This classification was only carried out in the summer in countries with consistent "ragweed" queries. Moreover, the patterns had to be consistent for 4 of the 5 years surveyed. We did not compare the results of the 5 -year trends with those of 1 year or less, since the goal of the paper was not to define whether GT can be used as a sentinel, but to assess whether there are differences between terms across the 15 countries of interest.

\section{Results}

GT Terms Reporting Symptoms or the Pollen Season

The 5-year GT are presented in Figures 1 and 2 and in online supplementary Figures 1 and 2. When the term "allergy" was searched, different patterns could be observed at the country level. In Austria and France, the term "ragweed" was largely used, but this did not always correlate with "allergy" peaks during the corresponding "ragweed" search peak. In Canada, Croatia, Hungary, and the USA, the term "ragweed" appeared to be less frequently searched, but there was a peak of "allergy" during the "ragweed" search peak. Using the GT translation, many countries did not have a ragweed peak. In Italy, we conducted the search for "Lombardia" (Lombardy) and the results were identical to the whole country (not shown).

\section{GT Translation of "Ragweed" into the Native Language}

We then searched for the term "ragweed" in the native language and major differences were observed in Croatia, Serbia and Slovenia (ambrozija), Hungary (parlagfü), and Romania (ambrozia; Fig. 2; online suppl. Fig. 2). In all of these countries, the searches were considerably different when the native language was used compared to the GT translation. This indicated that there were inadequate translations for some of the terms using GT. The terms in Cyrilic (Bulgaria) or Greek (Greece) were not searchable. For Germany, Italy, and the Netherlands, there were no differences between GT and manually translated terms. In all countries except Bulgaria, Greece, Italy, and the Netherlands, the ragweed pollen season could be demonstrated every year during the 5-year survey. The peak of queries for "ragweed" was sharper than that of "allergy."

\section{Seasonality of Queries in American and European \\ Countries}

The 5-year GT differed largely between countries when the terms "ragweed" (translated and native language), "pollen," "allergy," and "hay fever" were assessed (Fig. 1, 2; Table 2; online suppl. Fig. 1,2).

In 2 countries, it was impossible to assess GT accurately (Bulgaria and Greece). In 2 countries (Italy and the Netherlands) there was no increase in Google searches for the different terms during the summer. The term "pollen" was only searched for in the summer in Canada and Hungary. The term "ragweed" was searched for during the summer for all years in 12 countries. The term "allergy" was searched for between 4 and 5 years in 6 countries, twice or 3 times in 4 countries, and not at all in 5 countries. On the other hand, the terms "pollen" or "hay fever" were rarely searched for across the 5 years, and never in 14 and 13 countries, respectively.

Patterns of pollen seasons were compared for spring (March to June) and summer (July to September; Fig. 1, 2; Table 2; online suppl. Fig. 1, 2). For Canada, Croatia, and Romania, a similar pattern was found in spring and summer. In 8 countries, patterns differed between the spring and summer. "Allergy" was searched in all countries for all 5 years during the spring season and in 7 out of 11 countries during the summer season. However, 


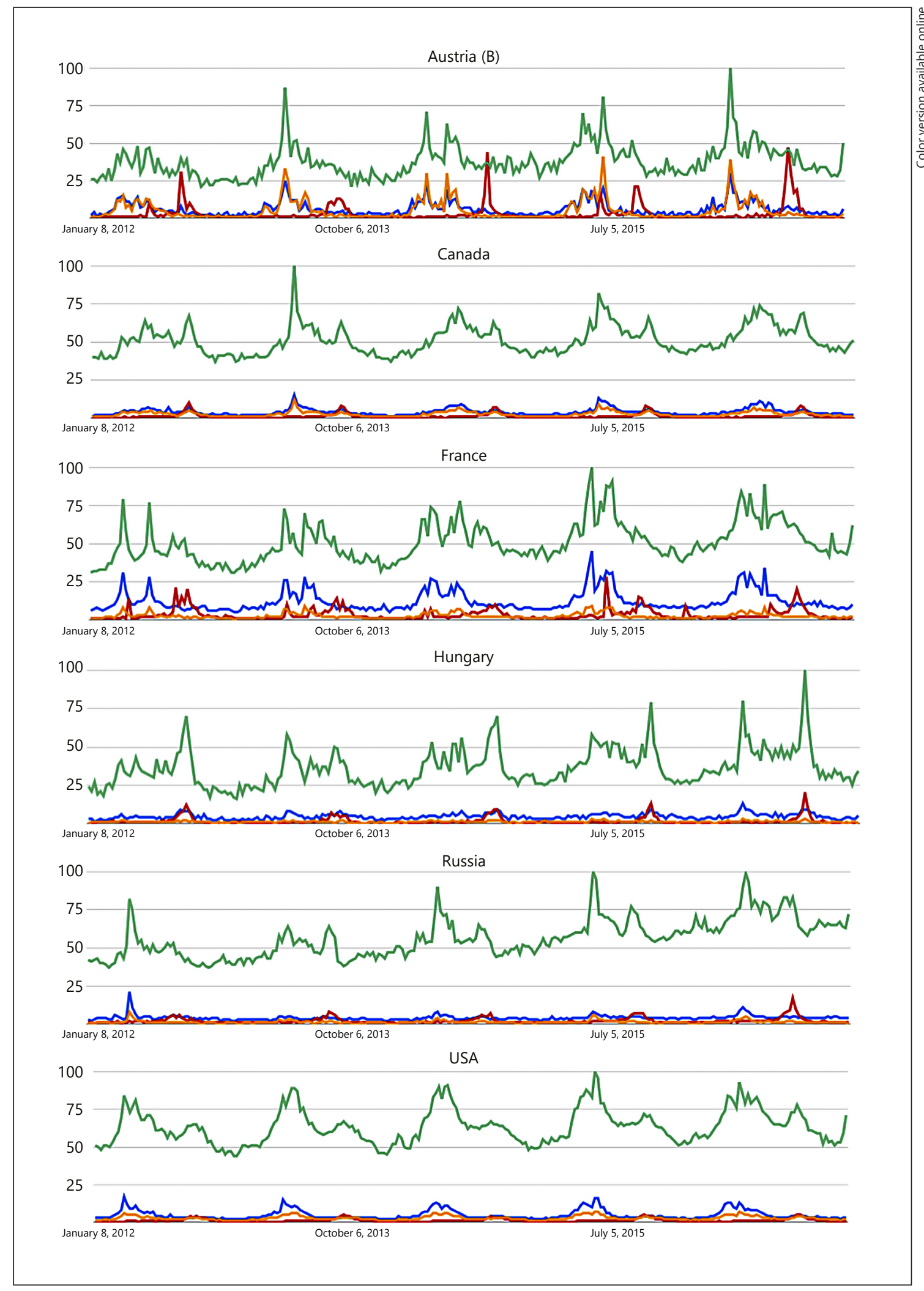

Fig. 1. Five-year GT for “allergy" (green lines), "hay fever” (blue), "pollen” (yellow), and "ragweed” (red) using GT-translated terms. 


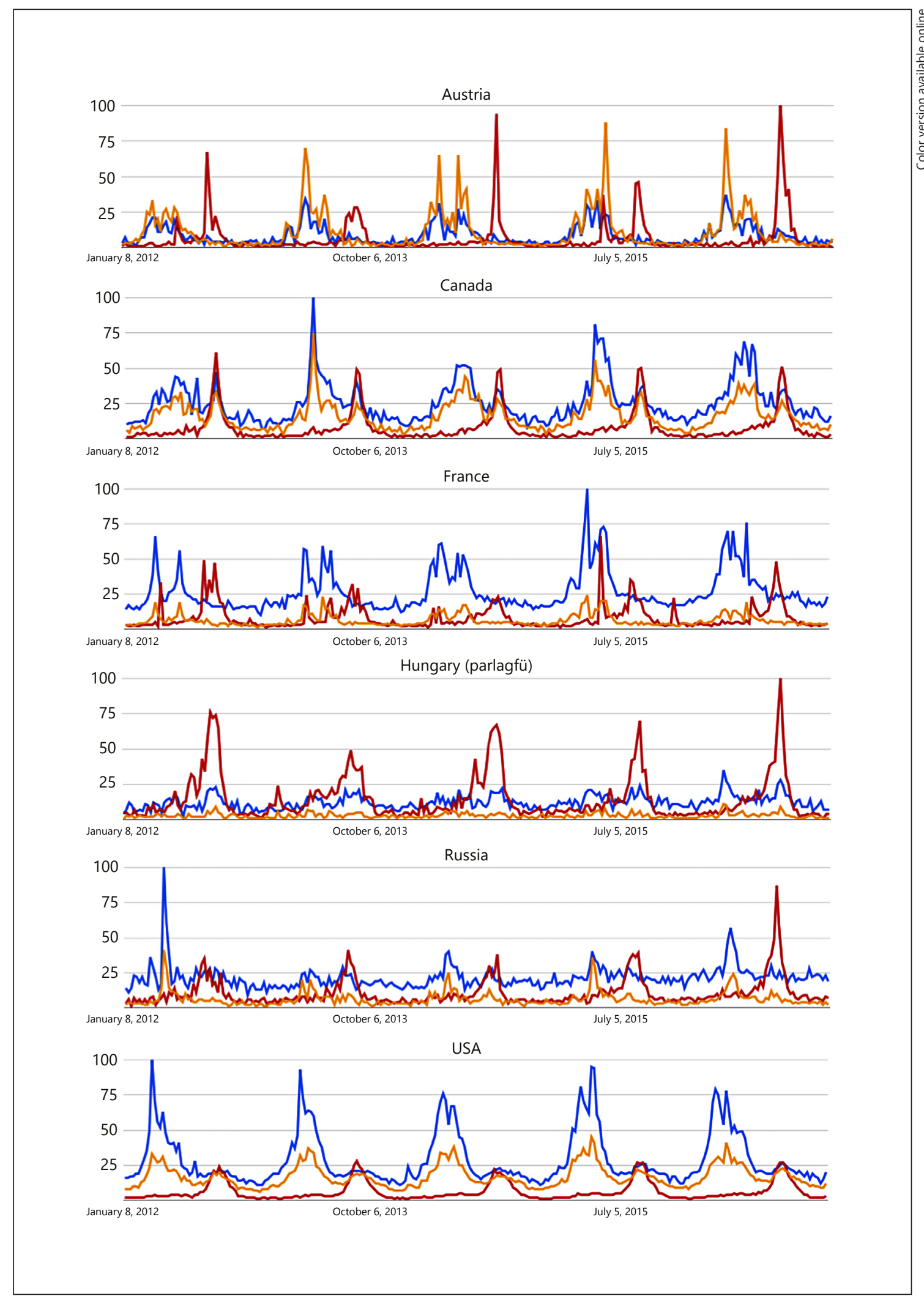

Fig. 2. Five-year GT for "hay fever" (yellow lines) "pollen" (blue), and "ragweed" (red) using GT-translated and manually translated terms. 
Table 2. GT in the spring and summer in countries with demonstrated seasons

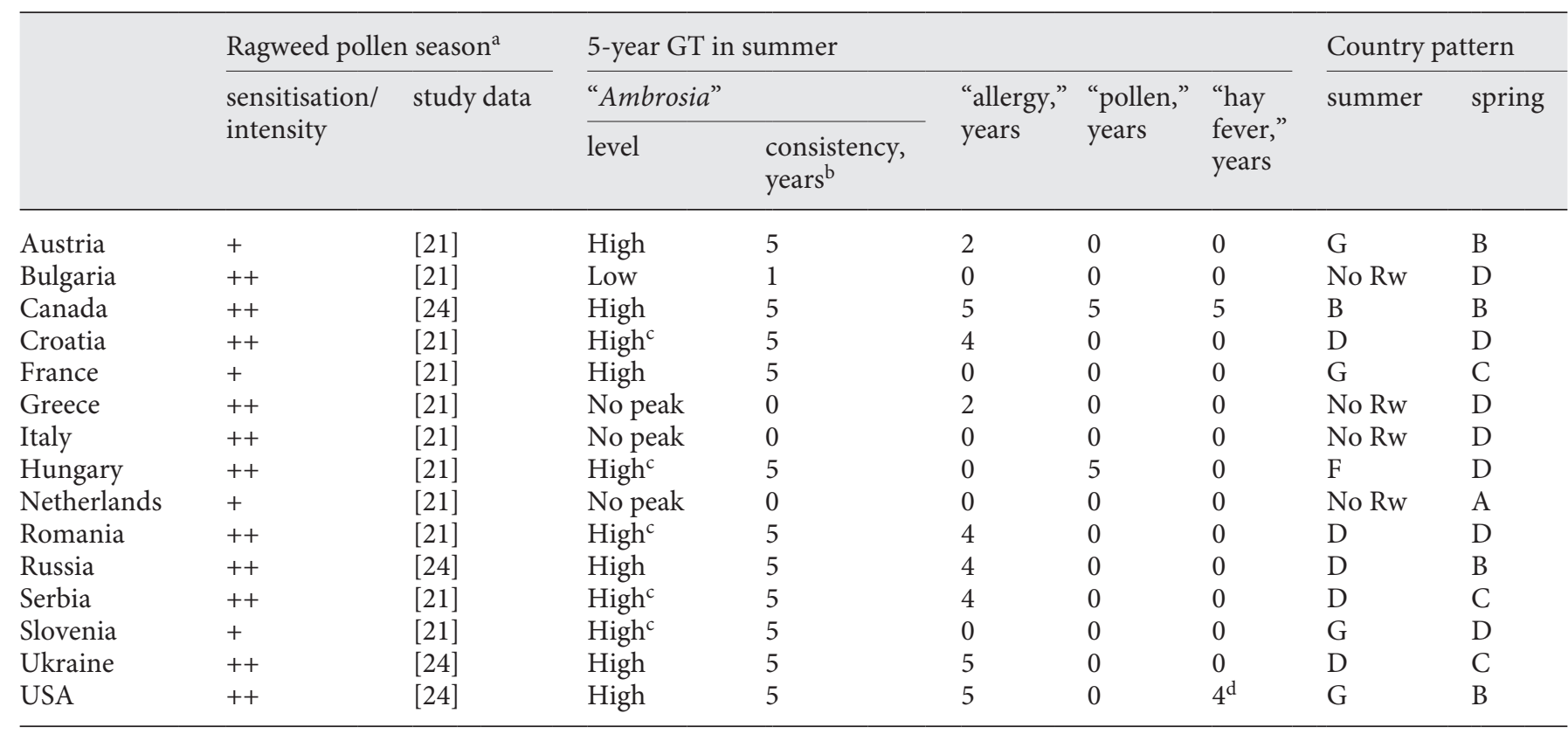

GT, Google Trends; Rw, ragweed.

a Determined by data obtained from the study by Essl et al. [21] or Prank et al. [24]. Data from Essl et al. [21] between 1986 and 2005. ,$+ 10 \%$ of the population of the country sensitised and $>1,000$ grains $/ \mathrm{m}^{3} ;++, 10 \%$ of the population of the country sensitised and $>2,500$ grains $/ \mathrm{m}^{3}$.

${ }^{b}$ Number of years of ragweed pollen/allergy peak over the 5-year monitoring period.

${ }^{\mathrm{c}}$ With the term in native language.

d Low peak.

except for Hungary, Serbia, and Ukraine, the peaks of queries were better identified in the spring than in the summer (Fig. 1; online suppl. Fig. 1).

\section{Discussion}

In the present study, searches for "ragweed" as a plant in 15 countries with a demonstrated pollen season show that the term can be used with the GT translation in 6 countries. Translations in the native language by GT were needed in 5 countries. In 4 countries, we were unable to identify a GT peak for "ragweed." However, when it exists, the peak for "ragweed" queries is easier to detect than the peak of "allergy" queries. There were apparent cultural differences in the knowledge or awareness of the terms between the spring and the summer pollen seasons in most countries. In countries with a high prevalence of ragweed pollen allergy, it is important to use "ragweed" (native language and GT translation) in addition to "al- lergy," "pollen," and "hay fever" to account for the pollen seasons using GT.

GT reflects the proportion of specific queries in relation to all queries. Although the terms were translated into the different languages using the automatic Google Translator, the reporting of GT differed widely between countries. We checked if the translation was appropriate for all countries and major differences appeared to be associated with translation issues for Croatia, Hungary, Romania, Serbia, and Slovenia. It appears that there are country-specific trends towards a different awareness and different use of allergy terms, which may represent cultural differences.

In the present paper we did not compare GTs with pollen counts as they are not available for all countries. We are currently analysing French data to make a direct comparison between all pollen seasons and GTs. For ragweed pollen, the time of occurrence of GTs is very similar across countries and in line with the ragweed pollen season. 
The selection of countries was based on a recent review in European countries [22] and the known ragweed pollen season in the former Yugoslavian countries [23], Canada, Russia, and Ukraine [24]. It is unclear how generalisable these findings are beyond these 15 countries under study.

The association between "pollen," "ragweed," and "allergy" searches differed between countries. However, "ragweed" was clearly identified in 12 of the 15 countries with a known ragweed pollen season. We then attempted to assess certain regions, such as Lombardy, and there was no difference. In Greece and the Netherlands, searches for "ragweed" were also not significant. Data were not interpretable in Bulgaria. These differences may be related to several possibilities: (i) the word "ragweed" is not the correct term used in the country; (ii) the translation of "ragweed" by GT is often incorrect (Croatia, Hungary, Romania, Serbia, and Slovenia); (iii) the pollen season did not induce severe symptoms in the country, which is possibly the case for Greece and the Netherlands - although countries were selected from a review assessing both the amount of ragweed pollen found in the atmosphere and the prevalence of the ragweed allergic population, severity was not reported [22]; (iv) an insufficient number of affected people in the country, and (v) cultural differences between countries cannot be appreciated. We have previously shown that such differences exist for AR and pollen, demonstrating country patterns [20]. Other possibilities include: (i) differences in media coverage of the ragweed pollen season, and (ii) known biases of GT, including the masking of some terms by a very high level of searches for 1 term relative to "ragweed." However, we used 2 different searches (with and without "allergy").

An important finding is represented by the differences in searches between the spring and summer pollen seasons. The terms "pollen" and "hay fever" were rarely searched for in the summer, suggesting some cultural differences between countries and/or awareness. It would be of interest to assess whether differences in the 2 seasons are associated with different treatment patterns. This can be done using the MASK sentinel network [1] or apps that can help monitor the treatment of AR in a large sample size observational study.

The May to June "ragweed" peaks detected in Austria and France are likely to represent awareness peaks since ragweed does not pollinate during these months. In particular, the 2015 May peak was associated with a paper on ragweed pollen and climate change that was published on May 25, 2015, and attracted substantial media interest [25]. In France, other peaks are found in May to June and may possibly be related to the information on Ragweed International Day, which is usually largely publicised. It is therefore of importance to correlate GT for "ragweed" with the expected pollen season. It is also important to correlate the pollen season with "ragweed" queries.

GT may be of interest in the differentiation of pollen seasons when they overlap, as is the case for many countries, including Austria, Canada, Hungary, and Slovenia. However, other tools may be used, such as Twitter [26].

The results of the current study will be used in the development of the MASK sentinel network [1]. MASK (MACVIA ["Contre les Maladies Chroniques pour un VIeillissement Actif”]-ARIA Sentinel Network) uses mobile technology to develop care pathways for the management of rhinitis and asthma by a multidisciplinary group or by patients themselves $[27,28]$. The scaling up strategy uses the recommendations of the European Innovation Partnership on Active and Healthy Ageing [29].

\section{Disclosure Statement}

J.B. reports personal fees from Almirall, Meda, Merck, MSD, Novartis, Sanofi-Aventis, Takeda, Teva, Uriach, AstraZeneca, Chiesi, GSK, and Menarini. R.M. reports personal fees from ALK, Allergopharma, Allergy Therapeutics, Bayer, FAES , Friulchem, GSK, Johnson\&Johnson, Meda, MSD, Nuvo, Servier, Stada, UCB, Klosterfrau, and Hexal, grants from ASIT Biotech, Leti, Optima, Ursapharm, BitopAG, and Hulka, grants and personal fees from Bencard and Stallergenes, grants, personal fees, and non-financial support from Lofarma and Novartis, and non-financial support from Roxall, Atmos, Bionorica, Ferrero, and Otonomy.

\section{Funding Sources}

This work was supported by European Union Development and Structural Funds.

References

\footnotetext{
1 Bousquet J, Schunemann HJ, Fonseca J, Samolinski B, Bachert C, Canonica GW, et al: MACVIA-ARIA Sentinel Network for allergic rhinitis (MASK-rhinitis): the new generation guideline implementation. Allergy 2015; 70:1372-1392.

2 Pfaar O, Bastl K, Berger U, Buters J, Calderon MA, Clot B, et al: Defining pollen exposure times for clinical trials of allergen immunotherapy for pollen-induced rhinoconjunctivitis - an EAACI Position Paper. Allergy 2017; 72:713-722.

-3 Caillaud D, Thibaudon M, Martin S, Segala C, Besancenot JP, Clot B, et al: Short-term effects of airborne ragweed pollen on clinical symptoms of hay fever in a panel of 30 patients. J Investig Allergol Clin Immunol 2014;24:249256.
} 
4 Csepe Z, Makra L, Voukantsis D, Matyasovszky I, Tusnady G, Karatzas K, et al: Predicting daily ragweed pollen concentrations using computational intelligence techniques over two heavily polluted areas in Europe. Sci Total Environ 2014;476-477:542-552.

$\checkmark 5$ Khwarahm NR, Dash J, Skjoth CA, Newnham RM, Adams-Groom B, Head K, et al: Mapping the birch and grass pollen seasons in the UK using satellite sensor time-series. Sci Total Environ 2017;578:586-600.

-6 Navares R, Aznarte JL: Predicting the Poaceae pollen season: six month-ahead forecasting and identification of relevant features. Int J Biometeorol 2017;61:647-656.

$>7$ Silva-Palacios I, Fernandez-Rodriguez S, Duran-Barroso P, Tormo-Molina R, Maya-Manzano JM, Gonzalo-Garijo A: Temporal modelling and forecasting of the airborne pollen of Cupressaceae on the southwestern Iberian Peninsula. Int J Biometeorol 2016;60:297306.

-8 Bastl K, Kmenta M, Pessi AM, Prank M, Saarto A, Sofiev M, et al: First comparison of symptom data with allergen content (Bet v 1 and $\mathrm{Phl} \mathrm{p} 5$ measurements) and pollen data from four European regions during 20092011. Sci Total Environ 2016;548-549:229 235.

>9 Buters JT, Weichenmeier I, Ochs S, Pusch G, Kreyling W, Boere AJ, et al: The allergen Bet $\mathrm{v} 1$ in fractions of ambient air deviates from birch pollen counts. Allergy 2010;65:850-858.

$>10$ Myszkowska D, Majewska R: Pollen grains as allergenic environmental factors - new approach to the forecasting of the pollen concentration during the season. Ann Agric Environ Med 2014;21:681-688.

$>11$ de Weger LA, Beerthuizen T, Hiemstra PS, Sont JK: Development and validation of a 5-day-ahead hay fever forecast for patients with grass-pollen-induced allergic rhinitis. Int J Biometeorol 2014;58:1047-1055.
12 Konig V, Mosges R: A model for the determination of pollen count using google search queries for patients suffering from allergic rhinitis. J Allergy (Cairo) 2014;2014:381983.

13 Willson TJ, Lospinoso J, Weitzel E, McMains $\mathrm{K}$ : Correlating regional aeroallergen effects on internet search activity. Otolaryngol Head Neck Surg 2015;152:228-232.

14 Zuckerman O, Luster SH, Bielory L: Internet searches and allergy: temporal variation in regional pollen counts correlates with Google searches for pollen allergy related terms. Ann Allergy Asthma Immunol 2014; 113:486-488.

15 Gaspar Marques J, Carreiro Martins P, Belo J Alves C, Paiva M, Caeiro E, et al: Pollen counts influence web searches for asthma and rhinitis. J Investig Allergol Clin Immunol 2016;26:192-194.

16 Willson TJ, Shams A, Lospinoso J, Weitzel E, McMains K: Searching for Cedar: geographic variation in single aeroallergen shows dose response in internet search activity. Otolaryngol Head Neck Surg 2015;153:770-774.

17 Oteros J, García-Mozo H, Viuf P, Galán C: Google trends, useful tool in airborne pollen detection. An Fac Med 2015;76:265-268.

18 Ginsberg J, Mohebbi MH, Patel RS, Brammer L, Smolinski MS, Brilliant L: Detecting influenza epidemics using search engine query data. Nature 2009;457:1012-1014.

19 Kang MG, Song WJ, Choi S, Kim H, Ha H, Kim SH, et al: Google unveils a glimpse of allergic rhinitis in the real world. Allergy 2015; 70:124-128.

20 Bousquet J, Agache I, Anto J, Bergmann K, Bachert C, Annesi-Maesano I, et al: Google Trends terms reporting rhinitis and related topics differ in European countries. Allergy 2017;72:1261-1266.
21 Essl F, Biro K, Brandes D, Broennimann O, Bullock JM, Chapman DS, et al: Biological flora of the British Isles: Ambrosia artemisiifolia. J Ecology 2014;103:1069-1098.

22 Lake IR, Jones NR, Agnew M, Goodess CM, Giorgi F, Hamaoui-Laguel L, et al: Climate Change and Future Pollen Allergy in Europe. Environ Health Perspect 2017;125;385-391.

23 Mehulic M, Mehulic K, Vuljanko IM, Kukulj S, Grle SP, Vukic AD, et al: Changing pattern of sensitization in Croatia to aeroallergens in adult population referring to allergy clinic during a period of 15 years. Coll Antropol 2011;35:529-536.

24 Prank M, Chapman D, Bullock J, Belmonte J, Berger U, Dahl A, et al: An operational model for forecasting ragweed pollen release and dispersion in Europe. Agric Forest Meteorol 2013;182/183:43-53.

25 Hamaoui-Laguel L, vautard R, Liu L, Solmon F, Viory N, Khvorostyanov D, et al : Effects of climate change and seed dispersal on airborne ragweed pollen loads in Europe. Nat Climate Change 2015;5:766-771.

26 Kmenta M, Zetter R, Berger U, Bastl K: Pollen information consumption as an indicator of pollen allergy burden. Wien Klin Wochenschr 2016;128:59-67.

27 Bousquet J, Addis A, Adcock I, Agache I, Agusti A, Alonso A, et al: Integrated care pathways for airway diseases (AIRWAYS ICPs). Eur Respir J 2014;44:304-323.

28 Bousquet J, Hellings PW, Agache I, Bedbrook A, Bachert C, Bergmann KC, et al: ARIA 2016: care pathways implementing emerging technologies for predictive medicine in rhinitis and asthma across the life cycle. Clin Transl Allergy 2016;6:47.

29 Bousquet J, Farrell J, Crooks G, Hellings P, Bel $\mathrm{EH}$, Bewick M, et al: Scaling up strategies of the chronic respiratory disease programme of the European Innovation Partnership on Active and Healthy Ageing (Action Plan B3: Area 5). Clin Transl Allergy 2016;6:29. 